\title{
Zehn Jahre auf der NATO-Agenda - Terrorismusbekämp- fung durch die Nordatlantische Allianz
}

\author{
Markus Kaim*
}

\begin{abstract}
After 9/11, NATO listed transnational terrorism among the major threats of the coming decade. Since then, structural as opposed to operational provisions remained partial, and NATO's four anti-terrorist operations have proven its instruments inefficient and its approaches inept. Confronted with different actors and threats, the US and several EU-members also perceive retaliation options differently. The NATO option took a back seat as its strategies appeared to duplicate existing ones on other levels. Today, coordination on ministerial levels, through an Assistant Secretary General, or a transatlantic advising board could contribute to a new multidimensional strategy. But success remains uncertain.
\end{abstract}

Keywords: NATO, transnational terrorism, counterterrorism, change of security policy institutions, security policy cooperation NATO, transnationaler Terrorismus, Terrorismusbekämpfung, Wandel sicherheitspolitischer Institutionen, sicherheitspolitische Zusammenarbeit

\section{Einführung}

I n den 60 Jahren ihrer Existenz hat sich das Aufgabenspektrum der Nordatlantischen Allianz stark ausdifferenziert: Dominierte bis 1989 die Funktion der kollektiven Verteidigung gegen den Warschauer Pakt die politischen Überlegungen und militärischen Planungen der NATO, so ist in den vergangenen 20 Jahren angesichts der veränderten sicherheitspolitischen Herausforderungen eine erhebliche Erweiterung der spezifischen Funktionen sowie des verwendeten Sicherheitsbegriffs festzustellen. Zwar bildet die kollektive Beistandsverpflichtung nach wie vor den Eckpfeiler des Bündnisses. Im politischen Alltag spielen heute jedoch ganz andere Aufgaben eine viel größere Rolle: Kriseninterventionen in ethno-territoriale Konflikte, die militärische Sicherung von Staatsaufbau oder nach dem Ende eines Bürgerkriegs oder eines staatlichen Zerfallsprozesses, der Kampf gegen die Proliferation von Massenvernichtungswaffen, die politisch-militärische Unterstützung des Transformationsprozesses zahlreicher Staaten, die nicht oder noch nicht Mitglied der NATO sind, der damit verbundene „Export" von Stabilität in die Nachbarschaft des Bündnisgebiets und die Einbettung der Allianz in das globale System von miteinander verflochtenen Sicherheitsorganisationen, um nur einige wenige neue Funktionen der NATO zu nennen.

Seit fast genau zehn Jahren steht die Herausforderung des transnationalen Terrorismus auf der Agenda der NATO. Im April 1999 hat die Allianz ihr nach wie vor gültiges Konzept verabschiedet, in dem darauf verwiesen wird, dass nach dem Ende des Ost-West-Konflikts die Sicherheitsinteressen der Allianz auch durch andere Risiken bedroht sein könnten als durch die Aggression staatlicher Akteure - etwa durch terroristische Angriffe. In dem Dokument formulierten die Mitglieder die Notwendigkeit, ihre Streitkräfte und die Infrastruktur der NATO diesen veränderten Rahmenbedingungen anzupassen. ${ }^{1}$ Während es bei den europäischen NATO-Mitgliedern zu diesem Zeitpunkt noch vergleichsweise wenig Erfahrung mit islamistisch inspiriertem Terrorismus gab, hatten die Vereinigten

PD Dr. habil. Markus Kaim ist Leiter der Forschungsgruppe Sicherheitspolitik in der Stiftung Wissenschaft und Politik (Berlin). Dieser Aufsatz wurde einem doppel-blinden Gutachterverfahren (peer-review) unterzogen..

1 Vgl. NATO, The Alliance's Strategic Concept (www.nato.int/docu/pr/1999/ p99-065e.htm)
Staaten bereits Berührung damit gehabt (World Trade Center 1993, Angriffe auf US-Botschaften in Nairobi und Dar es Salaam 1998). Mit den Anschlägen vom 11. September 2001 erfuhr die sicherheitspolitische Herausforderung durch den transnational agierenden, jüngst vor allem islamistisch inspirierten Terrorismus eine vollständige Neubewertung - auch aufgrund der erstmaligen Ausrufung des Verteidigungsfalls nach Artikel 5 des NATO-Vertrags - und genießt seitdem in den entsprechenden Bedrohungsanalysen der Allianz höchste Priorität.

Eine wichtige Funktion dieser „neuen NATO“ ist daher mittlerweile auch die Bekämpfung des transnationalen Terrorismus. Nicht zwangsläufig resultieren jedoch aus entsprechenden Bekundungen der NATO-Gremien auch effektive Schritte. Der Aufsatz analysiert daher die Frage der Leistungsfähigkeit der nordatlantischen Allianz in diesem noch jungen Politikfeld. In einem ersten Schritt werden die Terrorismusdefinition der NATO sowie die existierenden Maßnahmen der operativen und strukturellen Terrorismusbekämpfung analysiert. Anschließend werden die Probleme bei der Umsetzung der beschlossenen Maßnahmen bzw. deren Gründe untersucht. Abschließend werden die Entwicklungsmöglichkeiten dieses Politikfeldes innerhalb der nordatlantischen Allianz diskutiert.

\section{Terrorismusdefinition und Bedrohungsanalyse}

Auf dem Prager NATO-Gipfel im November 2002 verabschiedeten die Mitgliedstaaten ein Programm zur Bekämpfung des internationalen Terrorismus, in dessen Nachgang der NATOMilitärausschuss auch eine Definition dieses Phänomens vorlegte: »The unlawful use or threatened use of force or violence against individuals or property in an attempt to coerce or intimidate governments or societies to achieve political, religious or ideological objectives. ${ }^{2}$ Diese Definition orientiert sich an Festlegungen anderer internationaler Organisationen und ist in ihrer Breite genauso wenig kontrovers, wie die Gründe und Akteure, die das Gremium als mögliche treibende Kräfte hinter terroristischen Angriffen identifiziert hat: Religiöser Extremismus könne ebenso eine Quelle von Terrorismus sein wie - isoliert oder kombi-

2 Vgl. NATO, NATO's Military Concept for Defence against Terrorism, Annex A: Definitions (<www.nato.int/ims/docu/terrorism-annex.htm $>$ ). 
niert - wirtschaftliche, soziale, demografische und politische Faktoren, die häufig mit ungelösten politischen Konflikten und wirkmächtigen ideologischen Strömungen einhergingen. Dabei definiert das Programm den transnational operierenden Terrorismus als genauso gefährlich wie dessen Unterstützung durch Staaten. Zudem wird auf das Risiko hingewiesen, dass Terroristen in den Besitz von Massenvernichtungswaffen kommen könnten. Diese Prioritätensetzung hat auch die auf dem Rigaer NATO-Gipfel 2006 beschlossene Comprehensive Political Guidance bestätigt: Sie bezeichnet den Terrorismus und die Proliferation von Massenvernichtungswaffen als die beiden größten Bedrohungen der Allianz in den kommenden zehn bis 15 Jahren. ${ }^{3}$

\section{Maßnahmen der operativen und strukturellen Terrorismusbekämpfung}

Die Forschung, die sich mit Fragen der Terrorismusbekämpfung und ihrer Effektivität beschäftigt, verwendet weitgehend die analytische Unterscheidung in operative und strukturelle Maßnahmen der Terrorismusbekämpfung. Zu den operativen Maßnahmen werden jene gezählt, bei denen es in erster Linie darum geht, gegen bestehende und erkennbare terroristische Strukturen vorzugehen, einzelne Personen festzunehmen bzw. auszuschalten sowie die Planung und Durchführung von Anschlägen zu verhindern. Dabei ist auf der Akteursebene - innerstaatlich wie international - vor allem an Polizei und Geheimdienste, Anti-Terroreinheiten, das Militär, Strafverfolgungsbehörden und Gerichte, Zivil- und Katastrophenschutz, Zoll und Grenzschutz sowie Finanz- und Wirtschaftsbehörden zu denken. Unter dem Überbegriff der strukturellen Terrorismusbekämpfung werden hingegen diplomatische, sicherheits-, entwicklungs-, wirtschafts- und finanz-sowie kulturpolitische Maßnahmen verstanden, die darauf abzielen, die Entstehungsund Existenzbedingungen des Terrorismus, d.h. seine Ursachen bzw. sein ihn begünstigendes Umfeld, zu bekämpfen. Ziel ist dabei, terroristischen Gruppierungen und Netzwerken den sozialen und ideologischen Resonanzboden zu entziehen und auf diese Weise die Zahl ihrer Unterstützer und Sympathisanten zu reduzieren bzw. nicht weiter ansteigen zu lassen. ${ }^{4}$

Das auf dem Prager NATO-Gipfel im November 2002 vorgelegte Konzept zur Terrorismusbekämpfung (Military Concept for Defence Against Terrorism) weist der Allianz vier operative Kernaufgaben im Bereich der Terrorismusbekämpfung $\mathrm{zu}^{5}$ :

a. Unter dem Begriff des „Anti-terrorism“ werden jene, vorwiegend defensiv ausgerichteten Maßnahmen gebündelt, die die Verletzbarkeit von NATO-Truppen, von Bürgern der Mitgliedstaaten und materiellen Gütern gegenüber terroristischen Angriffen reduzieren sollen. Diese Maßnahmen bein-

3 NATO, Comprehensive Political Guidance, 9.11.2006 (<www.nato.int/docu/ basictxt/b061129e.htm>). Die Abschlusserklärung des Bukarester Gipfels im April 2008 ist mit ähnlichen Worten dieser Linie gefolgt: (<http://www.nato. int/docu/pr/2008/p08-049e.html>).

4 Vgl. dazu ausführlich Schneckener, Ulrich, Internationale Terrorismusbekämpfung im Spannungsfeld zwischen USA und Vereinten Nationen, in: ders. (Hrsg.): Chancen und Grenzen multilateraler Terrorismusbekämpfung, SWP-Studie S 14 (2007), S. 7-12 (S. 9).

5 Zu den Aushandlungsprozessen dieses Konzepts vgl. Deni, John R., Alliance Management: NATO's fight against Terrorism, in: Studia Diplomatica 60 (2007) 3, S. 137-156 (S.140ff.). halten eine begrenzte militärische Komponente als Reaktion auf terroristische Aktivitäten bzw. deren Eindämmung. Dabei ist die Einbindung nationaler ziviler Behörden notwendig. Diesem Bereich sind der Austausch geheimdienstlicher Informationen, die NATO-weite Standardisierung von Warnvorgaben und nationalen Verteidigungsrichtlinien genauso zuzuordnen wie die gegenseitige Unterstützung beim Schutz gegen Angriffe aus der Luft und von See. Dazu gehören ebenfalls Hilfsleistungen bei der Evakuierung von NATO-Staatsbürgern aus Gebieten, in denen eine erhöhte terroristische Gefahr besteht. Schließlich bündelt das Programm Defence against Terrorism (DAT) Forschungsaktivitäten, mit denen die NATO Technologien zum Schutz ihrer militärischen Einheiten sowie der Bürger ihrer Mitgliedstaaten entwickeln will. ${ }^{6}$ Die NATO-Mitglieder arbeiten in zehn Bereichen innerhalb dieses Programms an neuen Technologien, um unterschiedliche terroristische Angriffsformen abwehren zu können. Diese Aktivitäten umfassen zum Beispiel die Verbesserung der Schutzsysteme von Flugzeugen und Helikoptern gegen tragbare Raketen, den Schutz vor selbstgefertigten Bomben und die Verbesserung von Aufklärungstechnik. Das NATO Counter-terrorism Technology Development Programme (NCTDP) unterstützt die Mitgliedstaaten beim Aufbau dieser Kapazitäten. ${ }^{7}$

b. Für den Fall, dass ein terroristischer Angriff nicht verhindert werden kann, zielt das „Consequence Management “ darauf ab, die nationalen Sicherheitsbehörden dabei zu unterstützen, die Wirkung dieser Attacken abzufedern. Dies kann etwa durch die Bereitstellung von Einheiten geschehen, die auf die Bewältigung eines Angriffs mit Massenvernichtungswaffen (ABC-Schutz), die Wiederherstellung zerstörter Infrastruktur oder die Betreuung von Flüchtlingen spezialisiert sind. Im deutschen Sprachraum entsprechen Elemente des "Zivilschutzes“ weitgehend diesem Maßnahmenbündel. ${ }^{8}$

c. Unter der Kategorie „Counterterrorism“ subsumiert die Allianz all jene offensiven Schritte, die ebenfalls die Verwundbarkeit der oben genannten Ziele reduzieren, das heißt die Fähigkeiten terroristischer Gruppen signifikant schwächen bzw. eindämmen sollen. Darunter fällt eine Reihe disparat erscheinender Einzelaktivitäten: zum Beispiel die Entwicklung spezialisierter Anti-Terrorkräfte, gegenseitige logistische Unterstützung bei entsprechenden Einsätzen durch die Gewährung von Überflug- und Stationierungsrechten oder die kompensatorische Übernahme von Verpflichtungen in anderen Missionen, die durch den Anti-Terrorkampf einzelner Mitglieder vernachlässigt werden müssen. Die auf dem Prager Gipfel 2002 beschlossene und auf dem Rigaer Gipfel 2006 für einsatzfähig erklärte NATO Response Force lässt sich ebenfalls diesem Bereich zuordnen: Ihre 25.000 Soldaten sollen innerhalb von fünf Tagen einsatzbereit sein und eine Mission für mindestens 30 Tage übernehmen können. Zu ihrem Aufgabenkatalog gehört explizit auch Counterterrorism, etwa die Bekämpfung terroristischer Infrastruktur. Ferner haben die

6 NATO, Defence against Terrorism (DAT) programme (<www.nato.int/issues/ dat/index.html>).

7 Vgl. Daume, Herbert, Defence against Terrorism. Ein Aktionsprogramm der NATO, in: Europäische Sicherheit, 55 (2006) 3, S. 64-68.

8 Vgl. Skinner, Tony, Maximising Resilience. NATO CBRN Capabilities, in: Jane's Defence Weekly 45 (5 March 2008) 10, S. 24-26. 
NATO-Staaten auf dem NATO-Gipfel 2004 der Einrichtung einer ständigen Terrorist Threat Intelligence Unit zugestimmt, die beim NATO-Hauptquartier angesiedelt ist. Ihre Aufgabe ist es, jede Form von terroristischer Bedrohung zu analysieren und für einen besseren Informationsaustausch zwischen den NATO-Staaten zu sorgen.

d. Die vierte Rolle, die die NATO bei der multilateralen Terrorismusbekämpfung übernehmen will, ist die der »militärischen Zusammenarbeit«. Dabei wird davon ausgegangen, dass das institutionelle Primat bei der Terrorismusbekämpfung in den meisten Mitgliedstaaten bei den nationalen Sicherheitsbehörden liegt. Demzufolge sieht die NATO es als notwendig an, dass militärische Operationen nur in enger Abstimmung mit diesen erfolgen und kohärent mit nationalen Initiativen sein müssten. Außerdem strebt die NATO eine enge Koordination mit anderen internationalen Organisationen an, allen voran mit der EU und der OSZE.

In diesem Sinne spielt die Terrorismusbekämpfung auch eine wichtige Rolle innerhalb der existierenden NATO-Partnerschaftsformate. So haben die Mitglieder des Euro-Atlantischen Partnerschaftsrats (EAPC) auf ihrem Prager Treffen 2002 einen Aktionsplan gegen Terrorismus verabschiedet, der unter Bezug auf das Völkerrecht und existierende internationale Vereinbarungen detailliert Maßnahmen aufführt, zu denen sich die teilnehmenden Staaten im Bereich der Terrorismusbekämpfung verpflichten. ${ }^{9}$ Als Teil der EAPC-Vereinbarungen betreffen diese Beschlüsse auch das Partnership-for-Peace-Programm der NATO. ${ }^{10}$

Der NATO-Russland-Rat hat im Dezember 2004 ebenfalls einen gemeinsamen Aktionsplan zur Terrorbekämpfung beschlossen und in diesem Rahmen im Oktober 2006 in Italien eine erste gemeinsame Übung zum Katastrophenmanagement durchgeführt. ${ }^{11}$

Schließlich ist die Terrorismusbekämpfung eines der in der 2004 beschlossenen Istanbul Cooperation Initiative genannten sechs bilateralen Kooperationsfelder, in denen die Staaten des Golfkooperationsrates (GKR) mit der NATO zusammenarbeiten können.

Bei diesen Aktionsplänen bzw. Kooperationen handelt es sich jedoch um unverbindliche und auf der operativen Ebene sehr disparate Absichtserklärungen, deren Implementierung den einzelnen Staaten überlassen bleibt. Ein Kontroll- oder gar Sanktionsmechanismus, mit dessen Hilfe die Umsetzung der eingegangenen Verpflichtungen überprüft werden könnte, existiert nicht. Dementsprechend hat bisher keine Fortschreibung oder Überprüfung der Aktionspläne stattgefunden.

Konkret hat die NATO im Kontext der Terrorismusbekämpfung bislang vier Operationen durchgeführt:

Nach den Anschlägen des 11. September 2001 unterstützen die Mitgliedstaaten die Reaktion der Vereinigten Staaten im Sinne von Art. 5 des NATO-Vertrags. Dazu gehörte neben dem Austausch von Geheimdienstinformationen auch der Schutz von Einrichtungen der NATO-Partner sowie die bis Mai 2002 an-

\footnotetext{
9 NATO, Partnership Action Plan against Terrorism, 22. November 2002 (<www. nato.int/docu/basictxt/b021122e.htm>).

10 Vgl. dazu Howard, Russell D., Thinking Creatively in the War on Terrorism - Leveraging NATO and the Partnership for Peace Consortium, in: Connections, 4 (2005) 1, S. 1-8.

11 Siehe den Aktionsplan (<www.nato.int/docu/basictxt/b041209a-e.htm >).
}

dauernde Überwachung des Luftraums der USA (Operation Eagle Assist) durch Airborne Warning and Control System (AWACS)-Einheiten der Allianz. Zweitens schützt sie seit Oktober $2001 \mathrm{im}$ Rahmen der Operation Active Endeavor militärische und zivile Schiffe der NATO-Mitglieder im Mittelmeer. Gleichzeitig überwacht sie hier die internationale Schifffahrt auf eine mögliche Unterstützung terroristischer Aktivitäten. An dieser Mission nehmen auch verschiedene NATO-Partner, zum Beispiel Russland und die Ukraine, teil. ${ }^{12}$ Zum Dritten führt die NATO seit Sommer 2003 die International Security Assistance Force (ISAF) in Afghanistan, deren Aufgabe zwar nicht unmittelbar die Terrorismusbekämpfung ist. Ihr Mandat - nämlich die Stabilisierung Afghanistans und die Herstellung sowie Ausweitung von Staatlichkeit auf das gesamte Territorium des Landes auch gegen den Widerstand gewaltsam agierender Akteure - begreift die Allianz aber als einen Beitrag zur Terrorismusbekämpfung. Denn ein angestrebter Effekt des ISAF-Einsatzes ist es, terroristische Aktivitäten auf afghanischem Territorium dauerhaft zu unterbinden. Schließlich führte die NATO viertens auf dem Balkan kleinere Operationen zur Bekämpfung lokaler Terrorgruppen durch, die Verbindungen zum al-Qaida-Netzwerk unterhalten haben sollen. Diese Einsätze, deren Hauptziel die Eindämmung des Waffen- und Drogenschmuggels war, dienten durch ihre befriedende Wirkung auch den anderen laufenden NATO-Operationen auf dem Balkan.

Aufgrund des Charakters der NATO als militärische Allianz dominieren bei der Terrorismusbekämpfung die operativen Maßnahmen. Der einzige Teilbereich, der sich im weiteren Sinne der strukturellen Terrorismusbekämpfung zuordnen ließe, sind die bereits angesprochenen Partnerschaftsforen. Dies betrifft angesichts der geografischen Verortung des islamistischen Terrorismus im Nahen und Mittleren Osten ausschließlich die beiden auf dem NATO-Gipfel im Jahr 2004 eingerichteten Gremien: den Mediterranean Dialogue, der den Staaten des Mittelmeerraums ein Forum für politische Konsultationen und praktische Zusammenarbeit mit der NATO anbietet, und die Istanbul Cooperation Initiative, die einen Beitrag zur globalen und regionalen Sicherheit zu leisten sucht, indem sie den Staaten des GKR eine sicherheitspolitische Kooperation offeriert. Aus dieser Perspektive ließe sich auch der ISAF-Einsatz in Afghanistan der strukturellen Terrorismusbekämpfung zuordnen. Denn die Allianz legitimiert diese Stabilisierungs- und Wiederaufbaumission damit, dass sie erforderlich sei, um eine Rückentwicklung des Landes zu einem Hort des islamistischen Terrorismus zu verhindern.

\section{Probleme bei der Umsetzung der beschlos- senen Maßnahmen}

Mögliche externe Bedrohungen für das eigene Territorium sind naturgemäß der funktionale Referenzpunkt der Kooperation in der NATO: Als System kollektiver Verteidigung gegründet, war

12 Vgl. dazu ausführlich Cross, Sharyl, Russia's Relationship with the United States: NATO in the US-led Global War on Terrorism, in: The Journal of Slavic Military Studies, 19 (2006) 2, S. 175-192; Kelin, Andrej, Russia - NATO: Toward a New Stage of Interaction?, in: International Affairs (Minneapolis), 51 (2005) 1, S. 34-42; sowie Forsberg, Tuomas, Russia's Relationship with NATO: A Qualitative Change or Old Wine in New Bottles?, in: The Journal of Communist Studies and Transition Politics, 21 (2005) 3, S. 332-353. 
und ist es Teil des Selbstverständnisses der Allianz, einen Angriff auf das eigene Territorium abzuschrecken bzw. abzuwehren. Dabei wird in der Regel angenommen, dass es sich bei dem möglichen Angreifer ebenfalls um einen staatlichen Akteur mit einem entsprechenden Staatsterritorium handelt.

Angesichts der Herausforderung durch den islamistischen Terrorismus steht die NATO vor dem Problem einer Entterritorialisierung und damit verbundenen fehlenden Zurechenbarkeit: Bei den möglichen Angreifern handelt es sich nicht um klar identifizierbare staatliche Akteure, die geografisch eindeutig zu verorten wären. Selbst die Frage, ob es sich um einen externen Aggressor handelt, ist angesichts der Verwicklung einheimischer Bürger in Anschläge wie die von Madrid und London nicht ohne Weiteres zu beantworten. Dementsprechend sind viele militärische Instrumente der NATO für die Terrorismusbekämpfung schlicht ungeeignet, weil sie auf dem Prinzip der Territorialität basieren.

Daneben sehen sich die USA auf der einen und die europäischen Staaten der NATO auf der anderen Seite im Hinblick auf Terrorismus durchaus nicht denselben Akteuren und Gefahren gegenüber. Verkürzt formuliert: Die USA als globale Ordnungsmacht sind nahezu zwangsläufig mit anderen Herausforderungen dieser Art konfrontiert als die europäischen NATO-Mitglieder mit einem in der Regel auf Europa begrenzten Geltungs- und Wirkungsanspruch. Ferner unterscheiden sich die Bedrohungsperzeptionen ebenso wie die den NATOMitgliedern zur Verfügung stehenden Mittel zur Terrorismusbekämpfung. Konsequenterweise fallen daher ihre Antworten auf diese sicherheitspolitische Herausforderung nicht identisch aus. ${ }^{13}$ Diese Differenzen innerhalb der NATO kreisen um drei Bereiche:

a. Die USA betrachteten die Anwendung militärischer Gewalt nach den Anschlägen des 11. September als selbstverständlichen und notwendigen Bestandteil ihrer Anti-Terrorismus-Strategie. Dies schließt sowohl ein präventives als auch präemptives Vorgehen gegen staatliche Sponsoren terroristischer Gruppierungen sowie gegen die Proliferation von Massenvernichtungswaffen ein. ${ }^{14}$ Viele europäische NATO-Mitglieder sind bei der Bewertung der Effektivität dieses Instruments zurückhaltender und verweisen vor allem auf den transnationalen Charakter terroristischer Vereinigungen, gegen die militärische Instrumente nur begrenzt nutzbar seien.

b. Während die europäischen Staaten im Bereich der Terrorismusbekämpfung zumeist die Bedeutung internationaler Organisationen und deren Legitimation, die Beachtung des Völkerrechts und die Notwendigkeit eines größtmöglichen internationalen Konsenses betonen, stand die Bush-Administration den formellen Bindungen, die sich aus einem multilateralen Handeln für ihre Politik ergeben, eher ablehnend gegenüber. Stattdessen bevorzugte sie während eines Großteils ihrer Amtszeit unter Verweis auf die höher eingeschätzte Effektivität

13 Vgl. dazu ausführlicher Shapiro, Jeremy/Byman, Daniel, Bridging the Transatlantic Counterterrorism Gap, in: The Washington Quarterly, 29 (2006) 4, S. 3350 (35ff.).

14 Vgl. The National Security Strategy of the United States of America, March 2006, S. 18 u. 23: (<http://www.whitehouse.gov/nsc/nss/2006/nss2006. pdf $>$ ). die Kooperation in Koalitionen handlungsfähiger und -williger Akteure. $^{15}$

c. Die meisten europäischen NATO-Mitglieder erachten langfristig angelegte Strategien für notwendig, um die Wurzeln des Terrorismus zu bekämpfen. Dazu gehören Instrumente der Entwicklungszusammenarbeit, der Konfliktprävention und der Stabilisierung bzw. des Wiederaufbaus nach bewaffneten Konflikten. ${ }^{16}$ Ferner dominiert die Einschätzung, dass sich staatliche Akteure, die den Terrorismus unterstützen, durch positive Anreize zu einem kooperativen Verhalten bewegen lassen. Beide Ansätze hat die Bush-Administration zwar nicht prinzipiell zurückgewiesen. Sie hat jedoch die Ansicht vertreten, dass solche langfristig angelegten Strategien schnelle AntiTerrormaßnahmen nicht ersetzen können.

Schließlich funktioniert auch die von allen Seiten wiederholt als essentiell beschworene geheimdienstliche Zusammenarbeit nicht so gut, wie angesichts der Rhetorik zu erwarten wäre. Deutliche Abweichungen existieren im Bereich des Datenzugangs und des Datenschutzes, der Verwendbarkeit geheimdienstlicher Erkenntnisse vor Gericht und prinzipiell in der Abwägung von staatlichen Sicherheitsinteressen und bürgerlichen Freiheitsrechten.

Letztlich besitzt die NATO für die USA im Bereich der Terrorismusbekämpfung nur eine untergeordnete Bedeutung. Dies ist nicht zuletzt an der im September 2006 vorgelegten National Strategy for Combating Terrorism abzulesen, die die nordatlantische Allianz im Unterkapitel zur »Multilateralen Terrorismusbekämpfung «als Kooperationsforum der USA an nachrangiger Stelle hinter der Asia Pacific Economic Cooperation (APEC) und der Organisation Amerikanischer Staaten (OAS) aufführt. ${ }^{17}$ Seit Mitte der 1990er Jahre setzt Washington im Bereich der multilateralen Terrorismusbekämpfung eher auf die G 8 und die UN. ${ }^{18}$ Prinzipiell favorisieren die USA jedoch bilaterale Kooperationen, da diese unter dem Aspekt der Entscheidungsfindung und der Vertraulichkeit einfacher zu handhaben sind. Die BushAdministration war nach dem 11. September 2001 daher nicht bereit, ihre politischen Prioritäten einem als quälend lang empfundenen Entscheidungsprozess innerhalb der Allianz zu unterwerfen, dessen Ausgang und Effektivität ungewiss schienen. Die unterschiedlichen Positionen innerhalb der NATO sollten eine schnelle und entschlossene Reaktion der USA auf die Anschläge in New York und Washington nicht verhindern. ${ }^{19}$

Vor allem im Bereich der geheimdienstlichen Zusammenarbeit greifen die USA auf etablierte Kooperationsmuster zurück. Washington bevorzugt die traditionelle Zusammenarbeit zwi-

15 Vgl. Harvey, Frank P., Smoke and Mirrors: Globalized Terrorism and the Illusion of Multilateral Security, Toronto (u.a. 2004), S. 307-329.

16 Vgl. Rees, Wyn/Aldrich, Richard J., Contending Cultures of Counterterrorism: Transatlantic Divergence or Convergence?, in: International Affairs (London), 81 (2005) 5, S. 905-923 (913ff.)

17 Siehe National Strategy for Combating Terrorism, September $2006(<\mathrm{http}: / /$ www.whitehouse.gov/nsc/nsct/2006/nsct2006.pdf >), S. 19. Vgl. zur Zusammenarbeit der Vereinigten Staaten und der NATO bei der Terrorismusbekämpfung ausführlich De Nevers, Renée, NATO's international security role in the terrorist era, in: International Security 31 (Spring 2007) 4, S. 34-66.

18 Vgl. Badey, Thomas J., US Counter-terrorism: Change in Approach, Continuity in Policy, in: Contemporary Security Policy, 27 (2006) 2, S. 308-324 (313ff.).

19 Vgl. Bensahel, Nora, The Counterterror Coalitions. Cooperation with Europe, NATO, and the European Union, Santa Monica 2003, S. 15ff. Zur amerikanischen NATO-Politik vgl. Kaim, Markus, Pragmatismus und Grand Strategy. Die NATO-Debatte in den Vereinigten Staaten, SWP-Studie 31/06, Stiftung Wissenschaft und Politik (November 2006), 30 S. 
schen den angelsächsischen Ländern innerhalb (USA, Großbritannien) wie außerhalb der NATO (Australien, Neuseeland). ${ }^{20}$ Die bilateralen Kooperationsformen scheinen auf der operativen Alltagsebene auch innerhalb der NATO durchaus erfolgreich zu sein. Sie sind häufig von anderen, konfliktiven Ebenen der transatlantischen Beziehungen abgekoppelt und können gegebenenfalls sogar einen Beitrag dazu leisten, diese zu verbessern.

Die zunehmende Befassung der NATO mit der Terrorismusbekämpfung stößt aber nicht nur in Washington, sondern auch bei einigen europäischen Mitgliedern auf Vorbehalte: Eine Gruppe von Staaten unter französischer Führung argumentiert, dass es sich bei der Allianz um ein reines Militärbündnis handele und ihr Aufgabenkatalog daher nicht über die kollektive Verteidigung hinaus erweitert werden solle. Dies gelte umso mehr, als derartige Aktivitäten mit den Kompetenzen der Mitgliedstaaten im Bereich der inneren Sicherheit kollidieren. Die Aktivitäten der NATO drohten demnach, den Kern nationalstaatlicher Souveränität in diesem Politikfeld weiter auszuhöhlen, nachdem dies für viele europäische NATO-Mitglieder bereits durch die teilweise Vergemeinschaftung dieses Politikfelds im Rahmen der Europäischen Union der Fall ist.

Zwar ließe sich argumentieren, dass bis auf Frankreich die anderen NATO-Mitglieder auch durch die integrierte Kommandostruktur der Allianz bereits Teile ihrer Souveränitätsrechte abgegeben haben. Das Problem ist jedoch anders gelagert: Eine effektive Terrorismusbekämpfung durch die NATO wird nicht zuletzt dadurch erschwert, dass es sich dabei um ein Phänomen handelt, das mit der klassischen Trennung von Innenund Außenpolitik nicht zu bewältigen ist. Zugleich bestehen die NATO-Mitgliedstaaten jedoch weitgehend auf einer funktionalen Trennung dieser Politikfelder. Im Vergleich dazu liegt die Bereitschaft der EU-Mitglieder, in den Bereichen »domestic security « und »law enforcement « zu kooperieren, angesichts der Struktur der EU - einer hybriden Mischung intergouvernementaler und supranationaler Elemente - erheblich höher und die Effektivität der EU bei der Terrorismusbekämpfung ist entsprechend besser.

\section{Fazit und Perspektiven}

Es ist nicht überraschend, dass die NATO angesichts der skizzierten Beschränkungen im Vergleich zu anderen internationalen Organisationen im Bereich der Terrorismusbekämpfung nur eine bescheidene Tätigkeitsbilanz vorzuweisen hat. Der Grund dafür ist, dass sie vergleichbare Aktivitäten anderer internationaler Organisationen bzw. einzelner Staaten zu duplizieren droht. Auch das individuelle Engagement der Mitgliedstaaten ist daher begrenzt. ${ }^{21}$ In diesem Sinne folgt die Allianz nicht den vorliegenden Empfehlungen der Vereinten Nationen, die eine Arbeitsteilung internationaler Organisationen gemäß deren spezifischen Kompetenzen empfohlen und von einer allum-

20 Vgl. Stuart, Douglas, NATO's Anglosphere Option. Closing the Distance between Mars and Venus, in: International Journal, 60 (2004-2005) 1, S. 171 187.

21 Vgl. Stuart, David, The War on Terrorism Would Not Be Possible without NATO: A Critique, in: Contemporary Security Policy, 25 (2004), S. 409-429 (422ff.). fassenden Strategie zur Terrorismusbekämpfung abgeraten haben. Mit ihrer rhetorisch breit angelegten, aber operativ dürftig unterfütterten Anti-Terrorismus-Strategie läuft die Allianz Gefahr, dass Erfolge ausbleiben und sie in diesem Politikfeld unglaubwürdig wird. ${ }^{22}$

In diesem Zusammenhang werden häufig zwei konkrete institutionelle Neuerungen diskutiert, die trotz der skizzierten Beschränkungen die Leistung der NATO im Bereich der Terrorismusbekämpfung verbessern sollen. Erstens könnte eine punktuelle Teilnahme der für die innere Sicherheit zuständigen Minister an speziellen Beratungen des dann erweitert tagenden NATO-Rats die Kluft zwischen der Behandlung äußerer und innerer Sicherheit innerhalb des Bündnisses reduzieren, indem die existierenden Bestrebungen koordiniert und die innen- wie verteidigungspolitischen Elemente einer multilateralen AntiTerrorismus-Strategie aufeinander abgestimmt würden. ${ }^{23}$ Einige andere Beobachter wollen diese Koordinationsfunktion nicht den NATO-Mitgliedern zuweisen, sondern schlagen die Ernennung eines entsprechenden Assistant Secretary General vor, der nicht nur die Bemühungen der Allianz intern koordiniert, sondern darüber hinaus auch die Maßnahmen der NATO mit denen der Europäischen Union, der Vereinten Nationen und anderer internationaler Organisationen. ${ }^{24}$

Zweitens könnte die NATO durch die Bildung einer informellen, hochrangigen Beratergruppe zu Fragen der Terrorismusbekämpfung, in der alle Mitglieder vertreten sein würden, dem Thema eine größere Sichtbarkeit und Dringlichkeit innerhalb der NATO verleihen und dadurch die Mitglieder zu einer engeren Kooperation veranlassen.

Zwei der zu Beginn ausgeführten Aufgabenbereiche, nämlich Anti-terrorism und Consequence Management berühren jedoch funktionale Bereiche, in denen das Primat des Handelns bei den nationalen oder sub-nationalen Sicherheitsbehörden liegt. Da die Mitgliedstaaten bzw. deren Verwaltungseinheiten diese Kompetenzen nicht aufweichen, geschweige denn mit der NATO zu teilen bereit sind, werden die Handlungsmöglichkeiten der Allianz in diesen Bereichen nicht über einen subsidiären Charakter hinauskommen.

Zwar scheinen die Aktivitäten der NATO im Bereich des Counter-terrorism auf den ersten Blick eine größere Wirksamkeit zu entfalten als in den anderen Aufgabenbereichen, doch laufen sie Gefahr - wie erwähnt - die Fähigkeiten einzelner Mitglieder und anderer internationaler Organisationen zu duplizieren. Die daraus resultierende Zurückhaltung der Mitglieder wird daran deutlich, wie problematisch sich die Generierung der für die NATO Response Force notwendigen Streitkräfte gestaltet.

Dennoch sehen einige Analytiker die Terrorismusbekämpfung keineswegs lediglich am Rand der NATO-Aufgaben angesiedelt, sondern verweisen - im Gegenteil - darauf, dass der Schutz der

22 Zu dieser skeptischen Einschätzung vgl. ebenso Bensahel, Nora, The Counterterror Coalitions.

23 Vgl. dazu Abshire, David u.a., Maximizing NATO for the War on Terror. Presidential Leadership Can Strengthen the Transatlantic Relationship by Defining and Pursuing Shared Homeland Security Interests, Center for the Study of the Presidency (2005), $47 \mathrm{~S}$.

24 Vgl. z.B. Clark, Richard A./ McCaffrey, Barry R. (Co-Chairs): NATO's Role in Confronting International Terrorism, The Atlantic Council of the United States / Policy Paper (2004), p.vii. 
NATO-Mitglieder bzw. ihrer Gesellschaften vor politischem Zwang zum Kernbereich des Artikels 5 gehöre. Daher führe der Kampf gegen eine terroristische Bedrohung die Allianz - wenn auch unter veränderten Vorzeichen - wieder an ihren Beginn zurück. Eine solche strategische Besinnung sei nicht nur unter sicherheitspolitischen Aspekten dringend geboten, sondern gewährleiste auch die notwendige Legitimation der NATO in den Mitgliedstaaten, die durch die Vielzahl von Auslandseinsätzen in den vergangenen Jahren nicht (mehr) hergestellt werden konnte. Unter dem Überbegriff »Transatlantic Homeland Defense « plädieren diese Beobachter daher für eine multidimensionale Strategie der Allianz, die Elemente diplomatischer, finanzieller, wirtschaftlicher, geheimdienstlicher und polizeilicher
Zusammenarbeit umfassen soll. Konkret soll die Zivilverteidigung in die Allianzdoktrin mit dem Ziel aufgenommen werden, das Territorium der NATO vor terroristischen Angriffen zu schützen. ${ }^{25} \mathrm{Ob}$ diese Vorschläge jedoch geeignet sind, die skizzierten strukturellen Beschränkungen zu überwinden, darf bezweifelt werden.

25 Vgl. Amas, Neyla u.a., Transatlantic Homeland Defense, Center for Technology and National Security Policy - National Defense University (Mai 2006), Special Report (<www.ndu.edu/inss/press/CTNSP-INSS/spl-rpt.pdf >); sowie Hamilton, Daniel S., NATO Summit I: In Area, or in Trouble, in: International Herald Tribune, 27.11.2006. Am stärksten wird diese Position von dem ehemaligen spanischen Ministerpräsidenten Aznar vertreten. Vgl. Aznar, Jose-Maria, NATO - An Alliance for Freedom, in: The RUSI Journal, 151 (2006) 4, S. 38-40.

\title{
Right or wrong: Debate in Russia on Conflict in Georgia
}

\author{
Diana Digol*
}

\begin{abstract}
The article presents a comprehensive overview of opinions in Russia on the August 2008 military conflict with Georgia. The positions presented include not only those of the political elite and political opinion-makers but also those of the cultural and social elite and of representatives of various ethnic groups involved in the conflict. The overview concludes by presenting results of public opinion polls. The period covered is between the commencement of hostilities on 8 August 2008 and the end of that year. The article is limited to Russia and does not cover the debates of neighbouring countries.
\end{abstract}

Keyworlds: Russia, Georgia, conflict, opinion

Russland, Georgien, Konflikt, Meinung

\section{Introduction}

$\mathrm{F}$ Irom the start of the conflict between Georgia and Russia the leading Western news agencies dedicated several reports daily to this conflict. The desire to understand the events was overwhelming. Experts on Russia and the Caucasus were in high demand by local and national news agencies. Initial assessments rapidly found their way into major European and American news channels and newspapers. The majority of the media blamed Russia for the developments and were looking for details.

What was happening in Russia? How were these events shown, interpreted, received and discussed within Russia, by Russians, Georgians, Ossetians and others? From the outset of the conflict, an increasingly intense and comprehensive debate took place on TV, radio and in print media. The mass media provided a platform to numerous persons of various political inclinations as well as apolitical individuals, intellectuals, academics and artist.

This article presents a comprehensive overview of the opinions in Russia on the August 2008 military conflict between Georgia and Russia. The premise is that analysing opinions of a variety of people would help to create a picture of these events from a Russian point of view. Telling this story, I speak with the voices of the people allowing myself to use summaries of their argu- ments. I conclude the overview by presenting the results of public opinion polls.

The opinions of the two highest ranking leaders of Russia, Dmitry Medvedev and Vladimir Putin are presented to delineate the official position of the political elite. The opinion of the official opposition (parties, other than the ruling one, represented in the parliament) is presented through the voices of Gennady Zyuganov, leader of the Communist Party of the Russian Federation (KPRF), Vladimir Zhirinovsky, the leader of the Liberal Democratic Party of Russia (LDPR) and Sergei Mironov, a leader of the party "A Just Russia" 1 . The position of other parties (unofficial opposition) is presented through the voices of Mikhail Kasyanov, leader of People's Democratic Union (PDU), Sergei Mitrokhin, a leader of the "Yabloko" party and Garry Kasparov, a leader of "United Civic Front".

The views of Natalia Narochnitskaya, from the Institute for Democracy and Cooperation, Stanislav Belkovskii, Director of the Institute for National Strategy and Alexandr Konovalov, President of the Institute of Strategic Evaluation are presented to delineate the position of Russian political scientists and analysts. The views of Russian intellectuals are presented by the journalist Vladimir Pozner and the writer Boris Strugatskii. In

\footnotetext{
Dr. Diana Digol is researcher at the Center for OSCE Research (CORE) at the Institute for Peace Research and Security Policy (IFSH), Germany.

1 In the current State Duma (October 2008), only these three parties, in addition to the governing party "United Russia", are represented.
} 